\title{
ON THE GROUP OF AFFINE TRANSFORMATIONS OF AN AFFINELY CONNECTED MANIFOLD ${ }^{1}$
}

\section{KATSUMI NOMIZU}

It has been proved by S. Myers and N. Steenrod that the group of all isometries of a Riemannian manifold is a Lie group. ${ }^{2}$ The purpose of the present note is to establish a similar theorem for the group of all affine transformations of an affinely connected manifold under the assumption of "completeness" which will be explained below. We do not know whether this assumption is really necessary, and with a hope of eliminating it in the future, we shall give a series of lemmas in the general case. The formulation and proof of the main theorem (Theorem 1) will be given in the last section.

The author would like to acknowledge valuable criticism from Professors Chern and Chevalley.

1. Preliminaries. Let $M$ be a differentiable manifold ${ }^{3}$ of dimension $n$ with an affine connection. According to Chern's formulation, the affine connection is given by a set of $n+n^{2}$ linear differential forms $\theta^{i}$ and $\theta_{j}^{i}(i, j=1,2, \cdots, n)$ on the bundle of frames $B^{*}$ of $M$ which satisfy the equations of structure of affine connection, in the following fashion. To a local coordinate system $\left(u^{i}\right)$ in $M$ there corresponds a system of local coordinates $\left(u^{i}, X_{i}^{k}\right)$ in $B^{*}$ defined by the condition that the $n$ vectors of any frame are given by $e_{i}$ $=\sum_{k} X_{i}^{k} \partial / \partial u^{k}(i=1,2, \cdots, n)$, where $\operatorname{det}\left\|X_{\imath}^{k}\right\| \neq 0$. Let $\left\|Y_{i}^{k}\right\|$ be the inverse matrix of $\left\|X_{i}^{k}\right\|$. Set

$$
\theta^{i}=\sum_{j} Y_{j}^{i} d u^{j} \quad \text { and } \quad \theta_{i}^{k}=\sum_{j} Y_{j}^{k}\left(d X_{i}^{j}+\sum_{l, m} \Gamma_{m l}^{j} X_{i}^{l} d u^{m}\right),
$$

where $\Gamma_{m l}^{j}$ are the so-called coefficients of the affine connection with respect to the local coordinates $\left(u^{i}\right)$. Then $\theta^{i}$ and $\theta_{i}{ }^{k}$ are linearly independent linear differential forms defined on the whole space $B^{*}$ and satisfy the following equations of structure:

Presented to the Society, October 25, 1952; received by the editors February 13, 1952.

${ }^{1}$ This work was originally done while the author was a research assistant at the University of Chicago, and has been revised while holding a Eugene Higgins Fellowship at Columbia University.

$2 \mathrm{~S}$. Myers and N. Steenrod, The group of isometries of a Riemannian manifold, Ann. of Math. vol. 40 (1939).

3 Differentiability means that of class $C^{\infty}$, though our results hold for weaker assumptions. On the other hand, we do not assume the second axiom of countability for $M$. 


$$
\begin{aligned}
& d \theta^{j}-\sum_{k} \theta^{k} \wedge \theta_{k}^{j}=\frac{1}{2} \sum_{l, m} P_{l m}^{j} \theta^{l} \wedge \theta^{m} \\
& d \theta_{i}^{j}-\sum_{l} \theta_{i}^{l} \wedge \theta_{l}^{j}=\frac{1}{2} \sum_{l, m} S_{i l m}^{j} \theta^{l} \wedge \theta^{m}
\end{aligned}
$$

where

$$
\begin{aligned}
\frac{1}{2} P_{l m}^{j} & =\sum_{i, p, q} Y_{i}^{j} X_{l}^{p} X_{m}^{q} T_{p q}^{i} \\
S_{i l m}^{j} & =\sum_{k, p, q, r} Y_{k}^{j} X_{i}^{p} X_{l}^{q} X_{m}^{r} R_{p q r}^{k}
\end{aligned}
$$

$T_{p q}^{q}$ and $R_{p q r}^{k}$ being the components of the torsion and curvature tensors respectively.

If $\phi$ is a differentiable homeomorphism of $M$ onto itself, it induces a differentiable homeomorphism of the tangent bundle $B$ and the bundle of frames $B^{*}$ onto themselves, which we shall denote by $D \phi . D \phi$ leaves the forms $\theta^{i}$ invariant. We shall call $\phi$ an affine transformation if $D \phi$ leaves the forms $\theta_{j}^{l}$ invariant.

For any element $b=(p, X)$ of $B$, where $p \in M$ and $X$ is an element of the tangent vector space $T_{p}$ of $p$, there exists a path (auto-parallel curve) $C_{b}(t)$, defined in $-\epsilon<t<\epsilon$ for some $\epsilon>0$, with origin $p$ and tangent vector at $p$ which is equal to $X$. With respect to a local coordinate system $\left(u^{i}\right)$ with origin $p, C_{b}(t)$ is expressed by a set of solutions of the system of equations

$$
d^{2} f^{i} / d t^{2}+\sum_{j, k} \Gamma_{j k}^{i} d f^{j} / d t \cdot d f^{k} / d t=0
$$

corresponding to the initial conditions

$$
f^{i}(0)=0, \quad\left(d f^{i} / d t\right)_{0}=\alpha^{i},
$$

where $X=\sum \alpha^{i} \partial / \partial u^{i}$. A parameter $t$ satisfying these conditions is uniquely determined and will be called the canonical parameter of the path $C_{b}$. We denote by $\Omega^{a}$ the set of points $b \in B$ such that the corresponding path $C_{b}(t)$ can be extended over the values of the canonical parameter $-\epsilon<t<a+\epsilon$ for some $\epsilon>0$. For each $b=(p, X)$ $\in \Omega^{1}$ we denote the point $C_{b}(1)$ by $\eta(b)=\eta(p, X)$ or $\eta_{p}(X)$. For each $p \in M$, let $\Omega_{p}^{a}$ be the set of elements $X \in T_{p}$ such that $b=(p, X) \in \Omega^{a}$.

LEMMA 1. For any $s>0$ and $a>0$, we have

$$
s \Omega_{p}^{a}=\Omega_{p}^{a / \bullet} \quad(p \in M) .
$$


Lemma 2. For each $a>0, \Omega^{a}$ is an open subset of $B$. For each $p \in M$, $\Omega_{p}^{a}$ is an open neighborhood of 0 in $T_{p}$.

LeMma 3. $b \rightarrow \eta(b)$ is a differentiable mapping of $\Omega^{1}$ into $M$.

These lemmas, whose setup is due to Chevalley, follow from the properties of solutions of $\left({ }^{*}\right)$. By a fundamental existence theorem in the theory of differential equations, there exist $\epsilon_{0}>0$ and $\delta_{0}>0$ such that the solutions $f^{i}(x, \alpha, t)$ of $\left(^{*}\right)$ with the initial conditions

$$
f^{2}(x, \alpha, 0)=x^{i}, \quad\left(d f^{i}(x, \alpha, t) / d t\right)_{t=0}=\alpha^{i},
$$

exist uniquely for $-\epsilon_{0}<t<\epsilon_{0}$, provided that $\left|x^{i}\right|<\delta_{0}$ and $\left|\alpha^{i}\right|<\delta_{0}$. Since we have $f^{i}(x, \alpha, s t)=f^{i}(x, s \alpha, t)$ for small $s>0$, we see that the solutions $f^{i}(x, \alpha, t)$ are defined for $-\epsilon<t<1+\epsilon$ for some $\epsilon>0$, provided that $\left|x^{i}\right|<\delta_{0}$ and $\left|\alpha^{i}\right|<\delta_{0} \epsilon_{0}$. We set $F^{i}(x, \alpha)=f^{i}(x, \alpha, 1)$ for $\left|x^{i}\right|<\delta_{0}$ and $\left|\alpha^{i}\right|<\delta_{0} \epsilon_{0} . F^{i}(x, \alpha)$ are differentiable functions in $x$ and $\alpha$. We also remark the following. Suppose that the path $f^{i}(x, \alpha, t)$ exists in a coordinate neighborhood for $-\epsilon<t<a+\epsilon$ for some $a>0$. Then for any $x$ and $\alpha$ which are sufficiently near $x_{0}$ and $\alpha_{0}$ respectively, a path $f^{i}(x, \alpha, t)$ exists for $-\epsilon<t<a+\epsilon$ and $f^{i}(x, \alpha, t)$ and $\left(d f^{i}(x, \alpha, t) / d t\right)_{t=a}$ are continuous in $x$ and $\alpha$. We shall now prove the above lemmas.

Proof of Lemma 1. Let $X \in \Omega_{p}^{a}$ and let $C_{b}(t),-\epsilon<t<a+\epsilon$, be the corresponding path. We define a path $C^{\prime}(t)$ by $C^{\prime}(t)=C_{b}(s t)$ for $-\epsilon / s$ $<t<a / s+\epsilon / s$. $C^{\prime}(t)$ has the tangent vector at $p$ which is equal to $s X$ and therefore $s X \in \Omega_{p}^{a / s}$. This being true for any $s>0$, we see that $s \Omega_{p}^{a}=\Omega_{p}^{a / s}$.

Proof of Lemma 2. It is sufficient to prove it for $a=1$. Let $b=\left(p_{0}, X_{0}\right) \in \Omega^{1}$ and let $C_{b}(t)$ be the corresponding path which is defined for $-\epsilon<t<1+\epsilon$ for some $\epsilon>0$. We cover the path by a finite number of coordinate neighborhoods $N\left(p_{0}\right), N\left(p_{1}\right), \cdots, N\left(p_{k}\right)$ such that $p_{1}=C_{b}\left(t_{1}\right), p_{2}=C_{b}\left(t_{1}+t_{2}\right), \cdots, p_{k}=C_{b}(1)$ and $p_{i+1} \in N\left(p_{i}\right)$ for each $i=0,1, \cdots, k-1$. Let $\left\{x^{1}, x^{2}, \cdots, x^{n}\right\}$ be a coordinate system in $N\left(p_{0}\right)$, and let $p_{0}=(0,0, \cdots, 0)$ and $X_{0}=\left(\alpha_{0}^{1}, \alpha_{0}^{2}, \cdots, \alpha_{0}^{n}\right)$. The path $C_{b}(t),-\epsilon^{\prime}<t<t_{1}+\epsilon^{\prime}$ for small $\epsilon^{\prime}>0$, lies in $N\left(p_{0}\right)$. By the remark we have made above, there exist $\delta_{1}, \delta_{2}>0$ such that for $q=\left(x^{1}, x^{2}, \cdots, x^{n}\right)$ and $Y=\left(\alpha^{1}, \alpha^{2}, \cdots, \alpha^{n}\right)$ with $\left|x^{i}\right|<\delta_{1}$ and $\left|\alpha_{0}^{i}-\alpha^{i}\right|<\delta_{2}$, a path with origin $q$ and tangent vector $Y$ at $q$ exists for $-\epsilon^{\prime}<t<t_{1}+\epsilon^{\prime}$. Furthermore, the point and the tangent vector of this path corresponding to $t=t_{1}$ are sufficiently near those of the path $C_{b}(t),-\epsilon^{\prime}<t<t_{1}+\epsilon^{\prime}$, if $q$ and $Y$ are sufficiently near $p_{0}$ and $X_{0}$ respectively. Using this argument backward starting from $N\left(p_{k-1}\right)$, we see that if $q$ and $Y$ are sufficiently near $p_{0}$ and $X_{0}$ respectively, the 
path corresponding to $(q, Y)$ can be defined for $-\epsilon<t<1+\epsilon$. This proves that the set $\Omega^{1}$ is open. The second statement is obvious.

Lemma 3 can be easily proved from what we have done so far.

Let $F^{i}(x, \alpha)$ be as before. It is easy to show that $\left(\partial F^{i}(x, \alpha) / \partial \alpha^{j}\right)_{\alpha=0}$ $=\delta_{j}^{\ell}$, that is, the differentiable mapping $(x, \alpha) \rightarrow(x, F(x, \alpha))$ has nonzero Jacobian at $(0,0)$. Hence there exists a neighborhood $W$ of 0 such that the above mapping is a differentiable homeomorphism of $W \times W$ onto its image $V$ which is an open set containing $(x, x)$, $x \in W$ (remark that $F(x, 0)=x$ ). We shall denote by $(x, y) \rightarrow(x$, $G(x, y))$ the inverse mapping which is also differentiable. We take a neighborhood $N$ of 0 such that $N \times N \subset V$. Then, for any $x, y \in N$ there exists $\alpha \in W$ such that $y=F(x, \alpha)$. We can take $N$ as small as we wish.

Now let $p_{0}$ be any fixed point of $M$. By taking a coordinate system $\left(u^{i}\right)$ with origin at $p_{0}$, we can consider $N$ as a neighborhood of $p_{0}$. For any two points $p=\left(x^{i}\right)$ and $q=\left(y^{i}\right)$ in $N$, there exists $\alpha \in W$ such that $F(x, \alpha)=y$, which means that there exists a tangent vector $X$ at $p$ such that $\eta(p, X)=q$. When $p$ is fixed, $q \rightarrow \eta_{p}^{-1}(q)$ is a differentiable homeomorphism of $N$ onto a neighborhood of 0 in $T_{p}$, which we shall denote by $N_{p}$. We call such a neighborhood $N$ a regular neighborhood in $M$. We have thus shown that any point of $M$ is contained in an arbitrarily small regular neighborhood.

Let $N$ be a regular neighborhood and $p, q$ two points in $N$. For any $X \in N_{p}, \eta(p, X)$ is a point in $N$ and hence is representable uniquely as $\eta(q, Y)$ for some $Y \in N_{q}$. We denote the mapping $X \in N_{p} \rightarrow Y \in N_{q}$ so defined by $\pi_{p q}$. It is a differentiable homeomorphism of $N_{p}$ onto $N_{q}$ with the inverse mapping $\pi_{q p}$.

LemMa 4. Let $N$ b'e a regular neighborhood and let $K$ be a compact set contained in $N$. Then the set $K^{*}=\bigcup_{p, q \in K}\left(p, \eta_{p}^{-1}(q)\right)$ is a compact set in $B$.

Proof. This is clear since the mapping $(p, q) \in N \times N \rightarrow\left(p, \eta_{p}^{-1}(q)\right)$ $\in B$ is continuous.

We have previously shown that the set $\Omega^{1}$ is an open set in $B$. We shall say that an affinely connected manifold $M$ is complete if $\Omega^{1}$ coincides with the whole $B$. If this is the case, the set $\Omega^{a}$ coincides with $B$ for every $a>0$ as we see from Lemma 1 . We see therefore that $M$ is complete if and only if every path $C_{b}(t), b \in B$, can be extended for any large value of the canonical parameter. A complete Riemannian manifold is complete in our sense when considered as an affinely connected manifold.

2. Affine transformations. An affine transformation of $M$ clearly 
maps any path into a path. From this fact we have

Lemma 5. Let $N$ be a regular neighborhood in $M$. Let $p_{0}$ be any fixed point in $N$. Any affine transformation $\phi$ can be expressed in $N$ by

$$
\phi(p)=\eta\left(\phi\left(p_{0}\right), D_{p_{0}} \phi\left(\eta_{p_{0}}^{-1}(p)\right)\right), \quad p \in N,
$$

where $D_{p_{0}} \phi$ denotes the mapping of $T_{p_{0}}$ into $T_{\phi\left(p_{0}\right)}$ which is induced by $\phi$.

LEMMA 6. Let $\phi$ be an affine transformation of $M$ such that $\phi\left(p_{0}\right)=p_{0}$ for a certain point $p_{0}$. If $D_{p_{0}} \phi$ is the identity mapping of the tangent space $T_{p_{0}}$, then $\phi$ is the identity transformation on $M$. In particular, if an affine transformation leaves a nonempty open set of $M$ pointwise fixed, it is the identity transformation.

Proof. If $\phi\left(p_{0}\right)=p_{0}$ and if $D_{p_{0}} \phi$ is the identity transformation of $T_{p_{0}}$, then $\phi$ is the identity mapping on a regular neighborhood $N$ of $p_{0}$ as we see from Lemma 5. For any point $q$ of $M$, we can take a finite number of regular neighborhoods $N\left(p_{i}\right)$ such that $P_{i+1} \in N\left(p_{i}\right)$ for each $i=0,1, \cdots, k-1$ and $p_{k}=q$. Then we see that $\phi$ is the identity transformation in each $N\left(p_{i}\right)$ and hence $\phi(q)=q$, which proves that $\phi$ is the identity transformation on $M$.

We shall say that a sequence of differentiable mappings $\phi_{n}$ of $M$ into itself converges strongly to a differentiable mapping $\phi$ of $M$ into itself if $\phi_{n}$ converges to $\phi$ over $M, D \phi_{n}$ to $D \phi$ over $B$, and $D D \phi_{n}$ to $D D \phi$ over the tangent bundle of $B$. Then the following is an almost immediate consequence of the definition of affine transformation.

Lemma 7. If a sequence of affine transformations of $M$ converges strongly to a differentiable homeomorphism $\phi$ of $M$ onto itself, then $\phi$ is an affine transformation.

We shall now prove

Lемма 8. Assume that $M$ is complete. Let $\phi_{n}$ be a sequence of affine transformations which satisfy the following conditions at a certain point $p_{0}$ of $M$ :

(1) $\phi_{n}\left(p_{0}\right)$ converge to a certain point $p^{\prime}$;

(2) $D_{p_{0}} \phi_{n}$ converge to a certain linear mapping $\Lambda$ of $T_{p_{0}}$ into $T_{p^{\prime} \cdot{ }^{4}}$

Then there exists a differentiable mapping of $M$ into itself which is the strong limit of $\phi_{n}$ on $M$.

Proof. Let $N$ be a regular neighborhood of $p_{0}$. We shall first prove that there exists a differentiable mapping of $N$ into $M$ which is the strong limit of $\phi_{n}$ on $N$. We define $\phi(p)=\eta\left(p^{\prime}, \Lambda\left(\eta_{p_{0}}^{-1}(p)\right)\right)$ for $p \in N$.

4 More precisely, the mappings $D_{p_{0} \phi_{n}}$ of $T_{p_{0}}$ into $B: X \in T_{p_{0}} \rightarrow\left(\phi_{n}\left(p_{0}\right),\left(D_{p_{0}} \phi_{n}\right)(X)\right)$ converge to the mapping $X \in T_{p_{0}} \rightarrow\left(p^{\prime}, \Lambda X\right) \in B$. 
The right-hand side is well defined since $M$ is complete. Since $\eta_{p_{0}}^{-1}$ and $\eta_{p^{\prime}}$ are differentiable mappings and since $\Lambda$ is a linear mapping, $\phi$ is a differentiable mapping of $N$ into $M$. It is also clear from definition that $D_{p_{0}} \phi=\Lambda$. Since $\phi_{n}$ are affine transformations, we have by Lemma 5

$$
\phi_{n}(p)=\eta\left(\phi_{n}(p),\left(D_{p_{0}} \phi_{n}\right)\left(\eta_{p_{0}}^{-1}(p)\right)\right) \quad \text { for } p \in N .
$$

We see thus that $\phi_{n}(p)$ converge to $\phi(p)$ for every $p \in N$. As for $D \phi_{n}$ and $D \phi$ we have

$$
D \phi_{n}=\left(D \eta_{\phi_{n}(p)}\right)\left(D_{p_{n}} \phi_{n}\right)\left(D \eta_{p_{0}}^{-1}\right)
$$

and

$$
D \phi=\left(D \eta_{p^{\prime}}\right)\left(D_{p_{0}} \phi\right)\left(D_{\eta_{p_{0}}}^{-1}\right)
$$

It is easy to show that $D \eta_{\phi_{n}(p)}$ converge to $D \eta_{p^{\prime}}$. Therefore we see that $D \phi_{n}$ converge to $D \phi$ over $N$. Similarly, we can prove that $D D \phi_{n}$ converge to $D D \phi$ over $N .^{5}$

In order to extend $\phi$ to the whole $M$, consider the totality of pairs ( $\left.U, \phi_{U}\right)$, where $U$ is an open set containing $N$ and $\phi_{U}$ is a differentiable mapping of $U$ into $M$ which is the strong limit of $\phi_{n}$ over $U$. By defining the partial order of extension as usual and using Zorn's lemma, we see that there exists a maximal $\left(U, \phi_{U}\right)$. If $U$ is not equal to $M$, we can find a regular neighborhood $N$ which has a point, say $p_{1}$, in common with $U$ and which does not entirely lie in $U$. Then the assumptions of our lemma being satisfied at this point $p_{1}$, we see that $\phi_{U}$ can be extended to a differentiable mapping of $U \cup N$ into $M$ which is the strong limit of $\phi_{n}$, which contradicts the maximality of $\left(U, \phi_{U}\right)$. This concludes the proof.

The following lemma can be easily proved.

LEMMA 9. Let $\phi_{n}$ and $\psi_{n}$ be sequences of affine transformations. If $\phi_{n}$ converge strongly to a differentiable mapping $\phi$ on $M$, then for any point $p$ and any neighborhood $V^{\prime}$ of $\phi(p)$, there exists a neighborhood $V$ of $p$ such that $\phi_{n}(V) \subset V^{\prime}$ for sufficiently large $n$. If $\phi_{n}$ and $\psi_{n}$ converge strongly to differentiable mappings $\phi$ and $\psi$ respectively on $M$, then $\phi_{n} \psi_{n}$ converge to $\phi \psi$ on $M$.

3. Theorem. We shall now formulate the main theorem. Let $M$ be an affinely connected manifold. Let $A(M)$ be the group of all affine transformations of $M$. If we introduce the compact-open topology

\footnotetext{
In these lines care must be taken of the meaning of the mappings and their differential mappings involved, as in the footnote 4.
} 
into $A(M)$, it is a topological transformation group consisting of differentiable homeomorphisms of $M$.

Theorem 1. If $M$ is complete, $A(M)$ is a Lie group.

In virtue of a theorem of S. Bochner and D. Montgomery, our theorem will be established if we prove that $A(M)$ is locally compact and that any element of $A(M)$ which leaves a nonempty open subset of $M$ pointwise fixed is the identity element. The last condition has been proved in Lemma 6 . We shall prove that $A(M)$ is locally compact.

Let $N$ be a regular neighborhood of any arbitrary but fixed point $p_{0}$ of $M$. Take two neighborhoods $U$ and $V$ of $p$ such that $\bar{U} \subset V$, $\bar{V} \subset N$, and $\bar{V}$ is compact and contained in a coordinate neighborhood. We shall prove that the closure $\bar{W}$ of the neighborhood $W=\{\phi$ $\left.\in A(M) \mid \phi(\bar{U}) \subset V, \phi^{-1}(\bar{U}) \subset V\right\}$ of the identity element of $A(M)$ is compact. Before this, we introduce a metric topology into $\bar{W}$ as follows: if $\phi, \psi \in \bar{W}$ are expressed by a set of functions $f^{i}(x)$ and $g^{i}(x)$ respectively with respect to any fixed coordinate system covering $V$, we set

$$
\rho(\phi, \psi)=\max _{1 \leqq i, j \leqq n}\left\{\left|f^{i}\left(p_{0}\right)-g^{i}\left(p_{0}\right)\right|,\left|\partial f^{i} / \partial x_{j}-\partial g^{i} / \partial x_{j}\right|_{p_{0}}\right\}
$$

and $\rho^{*}(\phi, \psi)=\rho(\phi, \psi)+\rho\left(\phi^{-1}, \psi^{-1}\right)$. Then $\rho$ and $\rho^{*}$ are metrics in $\bar{W}$ in virtue of Lemma 6 . It is not difficult to show that the $\rho^{*}$-topology in $\bar{W}$ is stronger than the topology in $\bar{W}$ which is induced by the compact-open topology of $A(M)$. Thus it is sufficient to prove that $\bar{W}$ is compact in the $\rho^{*}$-metric.

Let $\phi_{n} \in \bar{W}$. Since $\bar{V}$ is compact, the set $V^{*}=U_{p, q \in \bar{V}}\left(p, \eta_{p}^{-1}(q)\right)$ in the tangent bundle $B$ is compact by Lemma 4 . Since $\phi_{n}(\bar{U}) \subset V$, we see that $D_{p_{0}} \phi_{n}$ are mappings of $\bar{U}_{p_{0}}\left(=\eta_{p_{0}}^{-1}(\bar{U})\right)$ into $V^{*}$. $V^{*}$ being compact, we can take a subsequence such that $D_{p_{0}} \phi_{n_{\nu}}$ converge to a certain mapping which maps $\left(p_{0}, X\right)\left(X \in T_{p_{0}}\right)$ into $\left(p^{\prime}, \Lambda(X)\right) \in V^{*}$, where $p^{\prime}$ is a certain point in $\bar{V}$ and $\Lambda$ is a linear mapping of $T_{p_{0}}$ into $T_{p^{\prime}}$. We then know by Lemma 8 that there exists a differentiable mapping $\phi$ of $M$ into itself which is the strong limit of $\phi_{n_{y}}$. Since $\phi_{n_{\nu}}^{-1}(\bar{U}) \subset V$, we can take a subsequence again and assume that there exists a differentiable mapping $\psi$ of $M$ into itself which is the strong limit of $\phi_{n_{\nu}}^{-1} . \phi$ and $\psi$ being the strong limit of $\phi_{n}$, and $\phi_{n}^{-1}$ respectively, we see by Lemma 9 that $\phi \psi$ and $\psi \phi$ are the identity transformation, that is, $\phi$ and $\psi$ are differentiable homeomorphisms which are inverse

- S. Bochner and D. Montgomery, Locally compact groups of differentiable transformations, Ann. of Math. vol. 47 (1946). 
to each other. By Lemma $7, \phi$ is an affine transformation of $M$. Clearly $\phi \in \bar{W}$. We also see that $\phi$ is the limit of $\phi_{n}$ in the $\rho^{*}$-metric in $\bar{W}$. We have thereby proved that $\bar{W}$ is compact in the $\rho^{*}$-metric and hence in the compact-open topology of $A(M)$. This completes the proof of our theorem.

The following is evident.

THEOREM 2. Let $M$ be a Riemannian manifold and consider the affine connection which is induced by the metric. Then the group of all isometries $I(M)$ is a closed subgroup of $A(M)$.

University of Chicago and

Columbia University 